\title{
Recent patent applications in epigenetics
}

\begin{tabular}{|c|c|}
\hline Patent number & Description \\
\hline US8574832B2 & $\begin{array}{l}\text { A composition comprising an amplified DNA fragment, where the } \\
\text { fragment comprises a random nucleic acid sequence, a } 3^{\prime} \text { restric } \\
\text { tion site and a } 5^{\prime} \text { restriction site, where the } 3^{\prime} \text { and } 5^{\prime} \text { restriction } \\
\text { sites are identical; useful in the field of genomics, in particular, } \\
\text { genome-wide mapping and high-throughput sequencing from a } \\
\text { biological sample having a low number of cells. In some cases, } \\
\text { a chromatin map identifying gene locations may be determined } \\
\text { when using approximately } 1,000-100,000 \text { cells. }\end{array}$ \\
\hline
\end{tabular}

US8515150B2 Improved methods for identifying and producing induced pluripotent stem cells, comprising culturing fibroblasts (or other somatic cells); transfecting the fibroblasts (or other somatic cells) with one or more transcription factors; and reducing activity of one or more epigenetic regulators, wherein the fibroblasts (or other somatic cells) are induced to become pluripotent stem cells.

US8357666B2 A method for reprogramming a cell comprising exposing a cell to a short hairpin RNA (shRNA) construct that interferes with the expression of a gene that codes for a regulatory protein; inducing expression of a pluripotent gene; and selecting a cell, where differentiation potential has been restored to the cell.

US8323941B2 A method for monitoring eukaryotic histone demethylase activity, Harvard College involving contacting a eukaryotic histone demethylase protein with (Cambridge, MA, a histone peptide, where the histone peptide is lysine- or arginine- USA) methylated, and determining the methylation status of the histone peptide. Useful for gene regulation, in particular the modification of chromosome structure as a means of regulating transcription.

US8293494B2 A multiple-specificity demethylase complex comprising a Jumonji C University Medical (JMJC) domain-containing enzyme, capable of demethylating his- Center Freiburg tones; a method of providing said protein complex; and to the use of the complex in the medical field, in particular in the diagnosis and therapy of prostate cancer.

US8252761B2 A method of inhibiting, or at least reducing, undesirable cell proliferation and/or blockage of cell differentiation in target cells, comprising increasing the level of PCL2 in the target cells to a level that prevents cell proliferation. Also, a method of preparing a population of undifferentiated stem cells, comprising inhibiting the expression of $\mathrm{Pcl} 2$ in stem cells.

US8206935B2 A method for rapidly quantifying hydroxymethylated DNA by binding DNA to a plastic carrier followed by immunodetection of 5-hydroxymethylcytosine or hydroxymethylcytosine structure that is a marker of DNA hydroxymethylation.

US8076063B2 A method for detecting methylation in a population of doublestranded target nucleic acids, involving providing a population of double-stranded target nucleic acids labeled with purification tag, where the target nucleic acids comprise potentially methylated target sequences; cleaving the population of target nucleic acids with an enzyme; immobilizing the population of noncleaved target nucleic acids by the purification tag; and detecting the presence of the immobilized noncleaved target nucleic acids.

US8048634B2 Screening cancer, based on detecting the methylation rate of a tar- National Defense get gene in a test specimen, and using the methylation rate as the Medical Center screening index to determine the risk of having cancer, comprising (Taipei) detecting the methylation rate of $\mathrm{CpG}$ sequence in at least one target gene of the test specimen and determining whether there is cancer or precancerous pathological change or not in the specimen based on the methylation rate in the target gene, or using the methylation rate of target gene as prognosis marker.

\section{Assignee}

Massachuse

Technology

(Cambridge, MA

USA), General

Hospital Corp.

(Boston), Adli M,

Bernstein BE,

Mikkelsen TS

er Laboratory

mbridge, MA,

of Pittsburgh

(Pittsburgh, PA,

USA), Sammak PJ,

Mangoubi R,

Desai M, Erb TM,

Lowry N

Nupotential (Bato

Rouge, LA, USA)

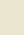

\author{
Research Institute
(Ottawa, Canada)
}

Sammak PJ,

10/13/2009

8/20/2013

Mangoubi R,

Desai M, Erb TM,

Lowry N

Inventor
Adli M,

Priority

application

Bernstein BE,

Mikkelsen TS

$2 / 3 / 2010$

Publication

date

$1 / 5 / 2013$

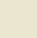

Shi Y, Shi Y

$12 / 16 / 2004$

$12 / 4 / 2012$

Eilertsen KJ,

$8 / 1 / 2005$

$1 / 22 / 2013$

Power RA, Rim JS

Ottawa Hospital

Stanford WL,

$6 / 10 / 2008$

$8 / 28 / 2012$

Wissmann M

$10 / 23 / 2012$

Walker E, Chang WY

Li W, Li J

Li W, Li J

$7 / 1 / 2010$

$6 / 26 / 2012$

Illumina (San Diego) Fan J-B

$2 / 7 / 2000$

$12 / 13 / 2011$ 